\title{
Clinical applicability of functional hemodynamic monitoring
}

Xaime García and Michael R Pinsky

\begin{abstract}
Recent interest in functional hemodynamic monitoring for the bedside assessment of cardiovascular insufficiency has heightened. Functional hemodynamic monitoring is the assessment of the dynamic interactions of hemodynamic variables in response to a defined perturbation. Accordingly, fluid responsiveness can be predicted in a quantities fashion by measuring as arterial pulse pressure variation and left ventricular stroke volume variation during positive pressure breathing or the change in cardiac output response to a passive leg raising maneuver. However, volume responsiveness, though important, reflects only part of the overall spectrum of functional physiological variables that can be measured to define physiologic state and monitor response to therapy. Dynamic tissue $\mathrm{O}_{2}$ saturation $\left(\mathrm{StO}_{2}\right)$ responses to complete stop flow conditions, which can be created by measuring hand $\mathrm{StO}_{2}$ and occluding flow with a blood pressure cuff, assesses cardiovascular sufficiency and microcirculatory blood flow distribution. Furthermore, these measures can be made increasingly more sensitive and specific if coupled to other "traditional" measures of organ perfusion, such as blood lactate levels.
\end{abstract}

\section{Introduction}

Recently, increased interest in a more proactive use of monitoring technologies has emerged, using the response to the measured variables to a defined stress to unveil the physiological state of the subject. This entire field when applied to the assessment of cardiovascular state is referred to as functional hemodynamic monitoring. Within this context, we can define functional hemodynamic monitoring as the assessment of the dynamic interactions of hemodynamic variables in response to a defined perturbation [1]. Such dynamic responses result in emergent parameters of these commonly reported variables that greatly increase the ability of these measures to define cardiovascular state and predict response to therapy. At the present time, the primary types of functional hemodynamic monitoring for which clinical trials have shown clinical usefulness are related to predicting volume responsiveness and identifying occult cardiovascular insufficiency (compensated shock). The primary form of this approach, predicting volume responsiveness, was reviewed recently in this journal, so will only be briefly described in this article. However, like any form of monitoring parameter, its sensitivity

\footnotetext{
* Correspondence: pinskymr@upmc.edu
Department of Critical Care Medicine, University of Pittsburgh, Pittsburgh PA

* Correspondence: pinskymr@upmc.edu
Department of Critical Care Medicine, University of Pittsburgh, Pittsburgh PA 15261, USA
} 15261, USA

\section{Springer}

(C) 2011 García and Pinsky; licensee Springer. This is an Open Access article distributed under the terms of the Creative Commons Attribution License (http://creativecommons.org/licenses/by/2.0), which permits unrestricted use, distribution, and reproduction in any medium, provided the original work is properly cited.

\section{Predicting volume responsiveness}

A primary resuscitation question is whether the patient will increase their cardiac output in response to intravascular volume infusion. Volume responsiveness has been arbitrarily defined as $\geq 15 \%$ in cardiac output in response to a $500-\mathrm{ml}$ bolus fluid challenge. Although the presence of fluid responsiveness in a subject does not equate for the need to give fluids, it does define that if fluids are infused cardiac output will increase. Many studies have validated, during recent years, the usefulness of some methods that give the clinician the ability to predict whether a patient is going to respond to the volume infusion as a primary step of the hemodynamic resuscitation. Some of the most commonly used 
methods are those based in changes in left ventricular output during positive pressure ventilation, such pulse pressure variation (PPV) and stroke volume variation (SVV). During the inspiratory phase of positive pressure ventilation, intrathoracic pressure increases passively, increasing right atrial pressure and causing venous return to decrease, decreasing right ventricular output, and after two or three heart beats, left ventricular output if both ventricles are volume responsive [2]. Thus, in preload-dependent patients cyclic changes in left ventricular stroke volume and its coupled arterial pulse pressure are seen and the magnitude of the changes is proportional to volume responsiveness. The associated SVV and PPV are quantified in various ways depending on whether these are measured by minimally invasive cardiac output monitors (e.g., PiCCO, LiDCO, FloTrac) or by direct examination of the pressure or flow profiles. In general both are defined as the ratio of the maximal minus the minimal values to the mean values, usually averaged over 3 or more breaths. Numerous studies have documented that a SVV $>10 \%$ or a PPV $>13-15 \%$ on a tidal volume of $8 \mathrm{ml} / \mathrm{kg}$ or greater is highly predictive of volume responsiveness [2-4].

Although powerful diagnostic tools, these parameters are highly dependent on the cyclic changes in intrathoracic pressure being regular and great enough to alter central venous pressure. Thus, tidal volumes of $\leq 6 \mathrm{ml} /$ $\mathrm{kg}$ or the imposition of variable spontaneous inspiratory efforts often result in false-negative PPV and SVV values. Moreover, all of these techniques assume a fixed heart rate, so in the setting of atrial fibrillation or frequent premature ventricular contractions, these measures become inaccurate. In these settings, one can always perform a passive leg raising maneuver [5].

Fluid therapy is one of the first steps in the goal-directed therapy in clinical guidelines of hemodynamic resuscitation of patients in shock [6,7]. Thus, an adequate assessment of fluid responsiveness should improve the therapy. A study conducted in high-risk surgery patients showed that volume loading guided to the goal of PPV minimization improves postoperative outcome and decreases length of hospital stay [8]. Patients in the interventional group received more fluid than the control group and had less postoperative complications, lower duration of mechanical ventilation, and shorter stay in the intensive care unit. Similarly, Pearse et al. [9] conducted a study to evaluate the effect of postoperative goal-directed therapy in postoperative high-risk patients. Targeting an oxygen delivery index $>600 \mathrm{~mL} / \mathrm{kg} /$ $\mathrm{min}$, they were able to reduce both postoperative complications and median duration of hospital stay.

\section{Identification of cardiovascular insufficiency}

Cardiovascular insufficiency is characterized by an inadequate $\mathrm{O}_{2}$ delivery relative to the metabolic demands. Shock can be, in the early stages, compensated by autonomic mechanisms, such regional vasoconstriction, in an attempt to maintain central blood pressure and vital organ perfusion above an anaerobic threshold. In this stage of compensated shock, microcirculatory measures, such as arterial pressure or cardiac output often are inside the range of values defined as normal and, therefore, insensitive as early predictors of subsequent decompensation due to the increased risk of tissue ischemia and subsequent development of multiorgan failure and death. However, microcirculation alterations in muscle and skin blood flow already occur during these early stages and measures of tissue cardiovascular reserve should be a sensitive early warning measure of impending cardiovascular collapse. Thus, a valid method to assess the microcirculatory status, such as the noninvasive measurement of tissue oxygen saturation $\left(\mathrm{StO}_{2}\right)$ when coupled with a functional hemodynamic monitoring test, such as the vascular occlusion test (VOT), may allow early identification of compensated circulatory shock and thus guide initial resuscitation efforts.

Noninvasive measurement of $\mathrm{StO}_{2}$ using near-infrared spectroscopy has been shown as a valid method to assess the microcirculation status, especially in septic and trauma patients. The absolute $\mathrm{StO}_{2}$ value has a limited discriminating capacity because $\mathrm{StO}_{2}$ remains within the normal range until shock is quite advanced. But the addition of a dynamic ischemic challenge, such as the VOT, improves and expands the predictive ability of $\mathrm{StO}_{2}$ to identify tissue hypoperfusion [10]. The VOT measures the effect of total vascular occlusioninduced tissue ischemia and release on downstream $\mathrm{StO}_{2} \cdot \mathrm{StO}_{2}$ is measured on the thenar eminence and transient rapid vascular occlusion of the arm by sphygmomanometer inflation to $30 \mathrm{mmHg}$ above systolic pressure is performed either for a defined time interval, usually $3 \mathrm{~min}$, or until $\mathrm{StO}_{2}$ declines to some threshold minimal value, usually $40 \%$. The deoxygenation rate $\left(\mathrm{DeO}_{2}\right)$ reflects the local metabolic rate and mitochondrial function, and the rate of reoxygenation rate $\left(\mathrm{ReO}_{2}\right)$ reflects local cardiovascular reserve and microcirculatory flow.

There is strong evidence for microcirculatory failure during shock to be a major component of the end-organ dysfunction seen. Such microcirculatory dysfunction can be characterized by oxygen shunting, vasoconstriction, thrombosis, and tissue edema. As a result of these combined microcirculatory events, the flow distribution within the tissue is impaired [11]. It has been shown that these microcirculatory alterations improve rapidly in septic shock survivors, whereas patients dying by organ failure have a lower percentage of perfused small vessels [12]. 
The hypothesis that the alterations in $\mathrm{VOT} \mathrm{StO}_{2}$ response are related to the outcome has been proved in patients with severe sepsis or septic shock by Creteur et al. [13]. Furthermore, when comparing hemodynamically stable patients without infection (controls) and healthy volunteers, these differences in the septic patients were striking. Using near-infrared spectroscopy $\mathrm{VOT} \mathrm{StO}_{2}$, they assessed the slope of increase in $\mathrm{StO}_{2}$ release as well as the difference between the maximum $\mathrm{StO}_{2}$ and the $\mathrm{StO}_{2}$ baseline $(\Delta)$. Both the slope of $\mathrm{ReO}_{2}$ and the $\Delta$ were significantly lower in septic patients than in control subjects and healthy volunteers. In the sample of septic patients, the slopes also were significantly lower in the ones who had cardiovascular insufficiency. $\mathrm{ReO}_{2}$ slopes were higher in survivors than in nonsurvivors and also tended to increase during resuscitation in survivors but not in nonsurvivors. Finally, the $\mathrm{ReO}_{2}$ slope was found to be a good predictor of ICU death, with a cutoff value of $2.55 \% / \mathrm{sec}$ (sensitivity $85 \%$, specificity $73 \%)$. These data confirm that the alterations in VOT $\mathrm{StO}_{2} \mathrm{ReO}_{2}$ are related more to the sepsis process itself and its severity than to mean arterial pressure or vasopressor agent's dose. Importantly, the magnitude of this $\mathrm{ReO}_{2}$ slope alteration is directly related to the septic disease and their presence in the first 24 hours of septic process and their persistence of delayed $\mathrm{ReO}_{2}$ slope is related to the patient's outcome. Still, if the $\mathrm{StO}_{2} \mathrm{ReO}_{2}$ does reflect inadequate tissue perfusion then it also should be sensitive of an impending cardiovascular insufficiency state (compensated shock) if matched with other static measures of tissue ischemia.

To address this issue, Guyette et al. [14] measured both the $\mathrm{VOT} \mathrm{StO}_{2}$ as baseline serum lactate, known to define existing cardiovascular insufficiency in trauma, in a cohort of trauma patients during the air transport to the Trauma Center. This study was designed to determine whether the $\mathrm{StO}_{2}$ measurement, including a VOT, was feasible in the prehospital environment and useful to predict in-hospital death and intensive care unit (ICU) admission. Not surprisingly, they did not find differences in baseline $\mathrm{StO}_{2}$ between survivors, nonsurvivors, and patients admitted to the ICU, and they showed significant differences in $\mathrm{DeO}_{2}$ and $\mathrm{ReO}_{2}$ slopes between survivors and nonsurvivors, as well as between patients who need ICU admission and patients who did not. Furthermore, only one of the five patient deaths in their sample had prehospital vitals signs that would have met the protocolized criteria for resuscitation (heart rate $>120 \mathrm{bpm}$, systolic blood pressure $<90 \mathrm{mmHg}$ ). Importantly, serum lactate alone was no better than lowest systolic pressure in predicting those in need of life-saving interventions or death, but if the baseline serum lactate was $>1.7 \mathrm{mmol} / \mathrm{dl}$ the $\mathrm{ReO}_{2}$ was $100 \%$ specific for the need of life-saving interventions. This study shows the usefulness of the microcirculation dynamic assessment in the early stages of the trauma injury, when cardiovascular insufficiency is not suspected with the macrocirculatory indexes, providing the possibility to start early the appropriate treatment and decide the inhospital disposition.

These studies show that microcirculation status, measured by a dynamic test, such the $\mathrm{StO}_{2} \mathrm{VOT}$, can be more accurate than the microcirculatory and static classic values assessing the cardiovascular insufficiency in patients with shock. A study conducted by Vallée et al. [15] proved the hypothesis that in septic patients when the goal of the resuscitation bundles (central venous oxygen saturation $\left.\left(\mathrm{SvcO}_{2}\right)>70 \%\right)$ has been already achieved, an index of tissue perfusion, such as the venous-to-arterial carbon dioxide difference $[\mathrm{P}(\mathrm{cv}-\mathrm{a})$ $\mathrm{CO}_{2}$ ], could be useful to identify those patients who still were inadequately resuscitated. Patients with $[\mathrm{P}(\mathrm{cv}-\mathrm{a})$ $\mathrm{CO}_{2}$ ] higher than $6 \mathrm{mmHg}$ had a lower cardiac index, a larger clearance of lactate, and a slower decrease of SOFA score in the first $24 \mathrm{~h}$ than patients with a $[\mathrm{P}(\mathrm{cv}-$ a) $\mathrm{CO}_{2}$ ] lower than $6 \mathrm{mmHg}$ [15].

The presence of cardiovascular insufficiency in patients with shock also can be assessed by metabolic products, such as lactate or strong ion difference (SID). The early presence of high levels of plasma lactate is associated with higher in-hospital mortality in patients presenting with circulatory shock $[16,17]$. Moreover, those patients who are able to decrease their lactate levels with the hemodynamic resuscitation procedures have a better outcome than those who are not. Thus, it would be reasonable to target lactate levels as an endpoint of the hemodynamic resuscitation interventions as a way to early know when a patient is being correctly resuscitated. A trial that focused on reaching a $\mathrm{SvcO}_{2}$ $>70 \%$ and lactate concentration $\leq 2 \mathrm{mmol} / \mathrm{L}$ by improving the oxygen delivery in postsurgical patients whose lactate levels were high or did not decrease showed a shorter hospital stay and lower morbidity [18]. A more recent study by Jones et al. [19] focused on comparing two early sepsis resuscitation protocols: one was designed to normalize central venous pressure (CVP), mean arterial pressure (MAP), and lactate clearance of at least $10 \%$, and another with $\mathrm{SvcO}_{2}$ as a goal, as well as CVP and MAP, showed no difference for in-hospital mortality.

In addition to the creation of lactate, tissue hypoperfusion due to cardiovascular insufficiency induces formation of anions as results of anaerobic metabolism and, finally, acidosis. The anion gap and, especially, the SID calculated as the difference between fully dissociated anions and cations, may help to evaluate those cases of metabolic disorders with acidosis adding the possibility to quantify the contribution of each metabolic disorder 
and to know the source of the acidosis. The approach, based on the principles described by Stewart [20], proposes the SID, as one of the three factors (together with the total weak acid difference and the $\mathrm{PaCO}_{2}$ ) that independently determine the $\mathrm{pH}$. It has been proven that the SID is useful to identify patients with major acidbase disturbances even when the standard base excess is normal [21]. Nevertheless, it has not been reflected in superiority against standard base excess predicting mortality, nor tested as goal of directed therapy in shock patients $[21,22]$.

\section{Conclusions}

Functional hemodynamic monitoring is the pluripotential approach to interpolation of physiological data in a proactive form. It has acquired a strong foothold in assessment of volume responsiveness for the management of the critically ill patient, showing a high applicability in shock resuscitation, but needs to be considered within the broader aspects of risk stratification to reach its full potential. Because early goal-directed therapy algorithms need to resuscitate to circulatory sufficiency, defining endpoints become as important as defining treatments to initiate. This field is rapidly expanding and has potential application for assessment of regional perfusion and function and across all acute care disciplines.

\section{Acknowledgements}

This work was supported in part by NIH grant HL067181.

\section{Authors' contributions}

XG wrote the original version of the manuscript after consultation with MRP. MRP defined the scope of the manuscript and wrote all the revisions of the manuscript to final form.

\section{Competing interests}

Xiame Garcia declares that he has no competing interests. Michael R. Pinsky is a consultant for Edwards LifeScieince, LiDCO Ltd, Cheetah Medical, Inc., has stock options with LiDCO Ltd., and has been paid to present a lecture by Edwards LifeScieinces, LiDCO Ltd and Hutchinson, Industries.

Received: 2 May 2011 Accepted: 25 August 2011

Published: 25 August 2011

\section{References}

1. Pinsky MR, Payen D: Functional hemodynamic monitoring. Crit Care 2005, 9:566-572.

2. Michard F, Boussat $S$, Chemla D, Anguel N, Mercat A, Lecarpentier $Y$, Richard C, Pinsly MR, Teboul JL: Relation between respiratory changes in arterial pulse pressure and fluid responsiveness in septic patients with acute circulatory failure. Am J Respir Crit Care Med 2000, 162:134-138.

3. Michard F, Teboul JL: Predicting fluid responsiveness in ICU patients: a critical analysis of the evidence. Chest 2002, 121:2000-2008.

4. Berkenstadt H, Margalit N, Hadani M, Friedman Z, Segal E, Villa Y, Perel A: Stroke volume variation as a predictor of fluid responsiveness in patients undergoing brain surgery. Anesth Analg 2001, 92:984-989.

5. Monnet $X$, Teboul JL: Passive leg raising. Intensive Care Med 2008, 34:659-663.
6. Rivers E, Nguyen B, Havstad S, Muzzin A, Knoblich B, Peterson E, Tomlanovich M: Early goal directed therapy in the treatment of severe sepsis and septic shock. N Engl J Med 2001, 345:1368-1377.

7. Dellinger R, Levy MM, Carlet JM, Bion J, Parker MM, Jaeschke R, Reinhart K, Angus DC, Brun-Buisson C, Beale R, Calandra T, Dhainaut JF, Gerlach H, Harvey M, Marini JJ, Marshall J, Ranieri M, Ramsay G, Sevransky J, Thompson BT, Townsend S, Vender JS, Zimmerman JL, Vincent JL: Surviving Sepsis Campaign: International guidelines for management of severe sepsis and septic shock: 2008. Intensive Care Med 2008, 34:17-60.

8. Lopes RL, Oliveira MA, Pereira VOS, Lemos IP, Auler JO Jr, Michard F: Goaldirected fluid management based on pulse pressure variation monitoring during high-risk surgery: a pilot randomized controlled trial. Crit Care 2007, 11:R100.

9. Pearse R, Dawson D, Fawcet J, Rhodes A, M Grounds M, Bennett D: Early goal-directed therapy after major surgery reduces complications and duration of hospital stay. A randomised, controlled trial. Crit Care 2005, 9 . R687-R693.

10. Gomez H, Torres A, Zenker S, Hyung K, Polanco P, Puyana JC, Pinsky MR: Use a non-invasive NIRS during vascular occlusion test to assess dynamic tissue $\mathrm{O} 2$ saturation response. Intensive Care Med 2008, 34:1600-1607.

11. De Backer D, Creteur J, Preiser JC, Dubois MJ, Vincent JL: Microvascular blood flow is altered in patients with sepsis. Am J Respir Crit Care Med 2002, 166:98-104.

12. Sakr Y, Dubois MJ, De Backer D, Creteur J, Vincent JL: Persistent microcirculatory alterations are associated with organ failure and death in patients with septic shock. Crit Care Med 2004, 34:1825-1831.

13. Creteur J, Carollo T, Soldati G, Buchele G, De Backer D, Vincent JL: The prognostic value of muscle $\mathrm{StO}_{2}$ in septic patients. Intensive Care Med 2007, 33:1549-1556.

14. Guyette F, Gomez H, Suffoletto B, Quintero J, Mesquida J, Kim HK, Hostler D, Puyana JC, Pinsky MR: Prehospital dynamic tissue $\mathrm{O}_{2}$ saturation response predicts in-hospital mortality in trauma patients. Crit Care Med 2009, 37(12 Suppl):A28.

15. Vallée F, Vallet B, Mathe O, Parraguette J, Mari A, Silva S, Samii K, Fourcade O, Genestal M: Central venous-to-arterial carbon dioxide difference: an additional target for goal-directed therapy in septic shock? Intensive Care Med 2008, 34:2218-2225.

16. Jansen TC, Van Bommel J, Mulder PG, Rommes JH, Schieveld SJM, Bakker J: The prognostic value of blood lactate levels relative to that of vital signs in the pre-hospital setting: a pilot study. Crit Care 2008, 12:R160.

17. Kyle J, Gunnerson KJ, Saul M, He S, Kellum JA: Lactate versus non-lactate metabolic acidosis: a retrospective outcome evaluation of critically ill patients. Crit Care 2006, 10:R228.

18. Pölönen $\mathrm{P}$, Ruokonen $\mathrm{E}$, Hippeläinen $\mathrm{M}$, Takala J: A prospective, randomized study of goal-oriented hemodynamic therapy in cardiac surgical patients. Anesth Analg 2000, 90:1052-1059.

19. Jones AE, Shapiro NI, Trzeciak S, Arnold RC, Claremont HA, Kline JA, the Emergency Medicine Shock Research Network (EMShockNet) Investigators: Lactate clearance vs central venous oxygen saturation as goals of early sepsis therapy. A randomized clinical trial. JAMA 2010, 303:739-746.

20. Stewart PA: Modern quantitative acid-base chemistry. Can J Physiol Pharmacol 1983, 61:1444-1461.

21. Boniatti MM, Cardoso PR, Castilho RK, Vieira SR: Acid-base disorders evaluation in critically ill patients: we can improve our diagnostic ability. Intensive Care Med 2009, 35:1377-1382.

22. Kellum JA: Disorders of acid-base balance. Crit Care Med 2007, 35:2630-2636.

doi:10.1186/2110-5820-1-35

Cite this article as: García and Pinsky: Clinical applicability of functional hemodynamic monitoring. Annals of Intensive Care 2011 1:35. 